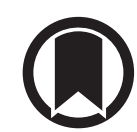

CrossMark

\title{
Fine particulate matter and out-of- hospital cardiac arrest of respiratory origin
}

\author{
To the Editor:
}

Exposure to ambient air pollution increases mortality and is a leading contributor to the global disease burden [1]. Epidemiological studies have elucidated a relationship between out-of-hospital cardiac arrests (OHCAs) and air pollutants, especially particulate matter (diameter $\leqslant 2.5 \mu \mathrm{m} ; \mathrm{PM}_{2.5}$ ) $[2,3]$. The causes of OHCA are broadly categorised as cardiac and non-cardiac [4]. A $10 \mu \mathrm{g} \cdot \mathrm{m}^{-3}$ increase in $\mathrm{PM}_{2.5}$ exposure yielded a $1.6 \%$ increase in the incidence of cardiac origin OHCA $[3,5]$. However, few studies on OHCAs of non-cardiac origin, including intrinsic respiratory diseases (COPD/pneumonia/asthma) are available. We examined the association between short-term exposure to $\mathrm{PM}_{2.5}$ and bystander-witnessed respiratory origin OHCAs, including eventual prognosis. We also investigated differences between $\mathrm{PM}_{2.5}$ exposure-related cardiac and respiratory origin OHCAs.

The All-Japan Utstein Registry, a prospective, nationwide, population-based registry that undertook Utstein-style data collection [6], was established (Fire and Disaster Management Agency). OHCAs registered between 1 January 2005 and 31 December 2016 were assessed and were presumed to be of cardiac origin unless a non-cardiac cause was evident. Measurements of ozone, nitrogen dioxide and sulfur dioxide in addition to $\mathrm{PM}_{2.5}$ concentrations in each air pollution monitoring station located in a distinct prefectural capital were obtained from the atmospheric environment database (National Institute for Environmental Studies). Data published by the Japan Meteorological Agency were used to evaluate the daily mean ambient temperature and relative humidity levels. Periods of influenza epidemic were defined as weeks in which the number of recorded cases were greater than the 90th percentile of the distribution during the study period.

The study design has been reported previously [5]. Briefly, a case-crossover design was used to examine the association between short-term $\mathrm{PM}_{2.5}$ exposure and OHCAs. The "case day" was defined as the day of OHCA occurrence; "control days" were selected using a time-stratified method [7]. We applied a conditional logistic regression model to estimate the odds ratios (with 95\% confidence intervals) for every $10 \mu \mathrm{g} \cdot \mathrm{m}^{-3}$ increase in $\mathrm{PM}_{2.5}$ concentrations at lag0-1 (mean $\mathrm{PM}_{2.5}$ concentrations on the case day and 1 day before). All analyses were performed using STATA 15.1 (Stata Corporation, College Station, TX, USA). A $p<0.05$ was considered statistically significant. This study was approved by the ethics committee of Kawasaki Medical School.

During 2005-2016 in Japan, 1423338 OHCAs were documented: 594791 were of non-cardiac origin; 243338 occurred during the $\mathrm{PM}_{2.5}$-monitoring period (April 2011-December 2016), of which, 72124 were bystander-witnessed. Of these, $21383 \mathrm{had}$ a respiratory aetiology. The mean \pm sD age was $80.6 \pm 13.8$ years (16598 were $\geqslant 75$ years, 10905 were men, and 12142 had OHCAs during cold seasons, i.e. November to April). Initial non-shockable rhythms (pulseless electrical activity/asystole) were detected in 20450 patients; 13271 patients received bystander resuscitation. The time from collapse to initial electrocardiogram (ECG) was $<10 \mathrm{~min}$ in 14125 respiratory origin OHCAs. The mean daily $\mathrm{PM}_{2.5}$ concentration was $13.9 \mu \mathrm{g} \cdot \mathrm{m}^{-3}$ by nationwide analysis. The prefecture-specific results for environmental factors were presented previously [5].

@ERSpublications

Particulate matter is a potential risk factor for out-of-hospital cardiac arrests (OHCAs) of respiratory origin. The percent increase in incidence of $\mathrm{OHCA}$ of respiratory origin is equivalent to that of $\mathrm{PM}_{2.5}$ exposure-related OHCAs of cardiac origin. http://bit.ly/3tDXym0

Cite this article as: Kojima S, Michikawa T, Matsui K, et al. Fine particulate matter and out-of-hospital cardiac arrest of respiratory origin. Eur Respir J 2021; 57: 2004299 [https://doi.org/10.1183/ 13993003.04299-2020]. 
a)

$$
\begin{array}{cc}
\text { Prefectures } & \text { Increase in } \\
n & \text { OHCA incidence } \\
& (95 \% \text { CI }) \# \%
\end{array}
$$

Total bystander-witnessed OHCAs of respiratory origin

Adjusted for $\mathrm{O}_{3}, \mathrm{NO}_{2}$ and $\mathrm{SO}_{2}$ at lag0-1

$$
\text { Age } \begin{aligned}
\quad & <75 \text { years } \\
& \geqslant 75 \text { years }
\end{aligned}
$$

Sex

$$
\text { Male }
$$

Female

Season Warm (May to October)

Cold (November to April)

b)

\section{Bystander-witnessed OHCAs}

Respiratory origin

Cardiac origin

Before propensity score matching

103189

After propensity score matching

c)

\section{Absence of ROSC}

1-month mortality

1-month survival with neurological impairment

19101

20869
$2.5(0.0-5.1)$

$2.2(-1.4-5.9)$

$2.8(-2.5-8.3)$

$2.4(-0.5-5.3)$

$3.6(0.0-7.3)$

$1.3(-2.2-5.0)$

$47 \quad 1.2(-2.5-5.0)$

$47 \quad 3.9(0.5-7.5)$

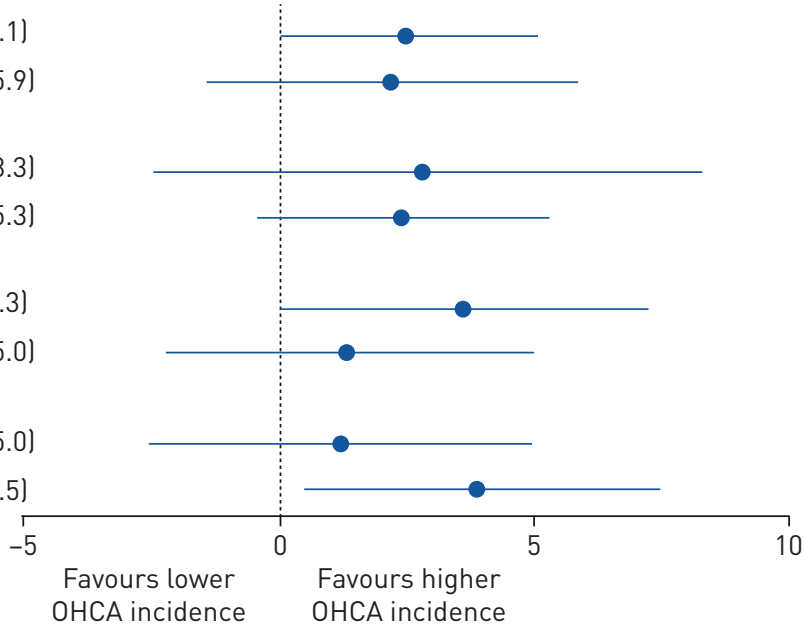

OHCA Increase in

patients OHCA incidence

n $\quad(95 \% \mathrm{Cl}) \# \%$

OHCA incidence

$21383 \quad 2.6(0.1-5.1)$

Favours lower

Favours higher

$\mathrm{OHCA}$ incidence

$2.5(0.0-5.1)$

$1.6(0.1-3.1)$

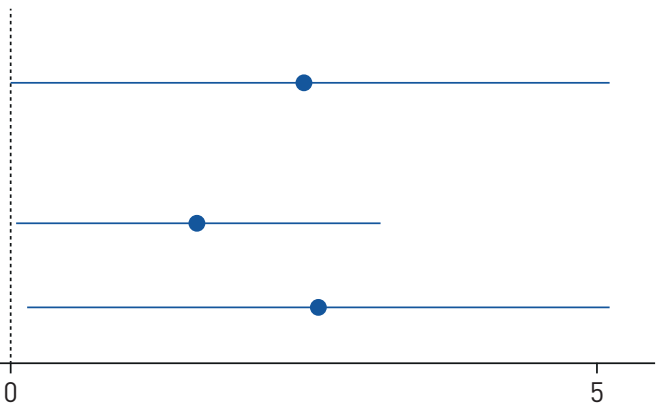

OHCA incidence

$\begin{array}{cc}\text { OHCA } & \text { Increase in } \\ \text { patients } & \text { OHCA incidence }\end{array}$

n $\quad(95 \% \mathrm{Cl}) \# \%$

$16832 \quad 3.1(0.3-6.1)$

$2.7(0.0-5.4)$

$2.4(-0.1-5.1)$

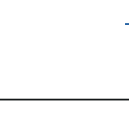

Favours no

association
Favours

association

FIGURE 1 a) $\mathrm{PM}_{2.5}$ (particulate matter with diameter $\leqslant 2.5 \mu \mathrm{m}$ ) exposure and total bystander-witnessed out-of-hospital cardiac arrests (OHCAs) of respiratory origin. OHCAs adjusted for ambient temperature/relative humidity at lag0-1 (mean $\mathrm{PM}_{2.5}$ concentrations on the case day and 1 day before) and incidence of influenza. b) Bystander-witnessed OHCAs of respiratory/cardiac origin affected by $\mathrm{PM}_{2.5}$ exposure before and after propensity score matching. The model included ambient temperature/relative humidity at lag0-1 (mean $\mathrm{PM}_{2.5}$ concentrations on the case day and 1 day before) and incidence of influenza. c) PM 2.5 exposure and poor outcomes following bystander-witnessed OHCAs of respiratory origin. ${ }^{\#}$ : odds ratio: percentage increase for every $10 \mu \mathrm{g} \cdot \mathrm{m}^{-3}$ increase in $\mathrm{PM}_{2.5}$ at lag0-1. ROSC: return of spontaneous circulation. 
Figure 1a demonstrates a stratified analysis of the sensitivity of the association between $\mathrm{PM}_{2.5}$ exposure and bystander-witnessed respiratory origin OHCAs (increase: $2.5 \%, 95 \%$ CI $0.0-5.1 \%$ ). Point estimates of percentage increases were higher in men (3.6\%, 95\% CI 0.0-7.3\%) and cold seasons (3.9\%, 95\% CI 0.5-7.5\%).

When the analysis was limited to patients with bystander-witnessed cardiac origin OHCAs during the same $\mathrm{PM}_{2.5}$ exposure period ( $\left.\mathrm{n}=103189\right)$, there was a significant association between $\mathrm{PM}_{2.5}$ exposure and OHCA incidence $(1.6 \%, 95 \%$ CI $0.1-3.1 \%)$. On comparing respiratory and cardiac origin OHCA incidences as related to $\mathrm{PM}_{2.5}$ exposure, heterogeneous patient backgrounds were observed. Following propensity score matching, 21383 (100\%) and 21383 (21\%) of respiratory and cardiac origin OHCAs were included in the analysis. For the date (month/year) and location (prefecture) of onset, we extracted the details of patients with cardiac origin OHCAs matched to those with respiratory origin OHCAs. Baseline covariates were well-balanced between the groups (absolute standardised difference $<10 \%$ ). The percent increase in cardiac origin OHCAs associated with increased $\mathrm{PM}_{2.5}$ concentrations was $2.6 \%$ (95\% CI 0.1 $5.1 \%$ ), which was equivalent to the percent increase in respiratory origin OHCAs associated with increased $\mathrm{PM}_{2.5}$ concentrations (figure $1 \mathrm{~b}$ ).

An initial shockable rhythm (ventricular fibrillation/pulseless ventricular tachycardia) was not significantly correlated with $\mathrm{PM}_{2.5}$ exposure and had a wide confidence interval because the rate of patients was only $1.8 \%$. However, there was a trend of an association between $\mathrm{PM}_{2.5}$ exposure and a non-shockable rhythm (2.4\%, 95\% CI $-0.2-5.1 \%)$. A time-stratified analysis was performed and the non-shockable rhythm detected $\geq 10(2.8 \%, 95 \%$ CI $-1.6-7.5 \%)$ or $<10 \min (2.2 \%, 95 \%$ CI $-1.0-5.4 \%)$ after the period from collapse to initial ECG did not correlate with $\mathrm{PM}_{2.5}$ exposure $(\mathrm{p}=0.90)$.

We examined the association of $\mathrm{PM}_{2.5}$ exposure with poor prognosis in patients with respiratory origin OHCAs (figure 1c). Increased $\mathrm{PM}_{2.5}$ concentrations were detected in patients who experienced an absence of return of spontaneous circulation $(3.1 \%$, 95\% CI $0.3-6.1 \%)$, 1 -month mortality $(2.7 \%$, 95\% CI $0.0-$ $5.4 \%)$, or 1-month survival with neurological impairment $(2.4 \%, 95 \% \mathrm{CI}-0.1-5.1 \%)$.

Our findings suggest that short-term $\mathrm{PM}_{2.5}$ exposure is associated with bystander-witnessed respiratory origin OHCAs in Japan. We had a large sample size (nationwide data of $>20000$ bystander-witnessed respiratory origin $\mathrm{OHCAs}$ ) for point estimates of a $\mathrm{PM}_{2.5}-\mathrm{OHCA}$ association, which may increase accuracy.

A previous meta-analysis reported that mortality was higher due to respiratory rather than cardiac causes related to $\mathrm{PM}_{2.5}$ exposure [8]. However, this association cannot be simply accepted, given the differences in patient background between respiratory versus cardiac origin OHCAs. Propensity score matching demonstrated the percent increase in incidence of respiratory origin OHCA associated with increased $\mathrm{PM}_{2.5}$ concentrations was equivalent to that of cardiac origin OHCA associated with increased $\mathrm{PM}_{2.5}$ concentrations, suggesting that the initial $\mathrm{PM}_{2.5}$-induced mechanism associated with the underlying respiratory or cardiac disease may be identical, followed by cardiopulmonary deterioration and OHCA. $\mathrm{PM}_{2.5}$ can be inhaled deeply into the small airways/alveoli of the lungs and may increase and sustain oxidative stress throughout the respiratory tract and at a systemic level to induce inflammation $[9,10]$. In the lungs, particulate matter may induce alveolar inflammation, thereby activating cellular and molecular events, aggravating pre-existing pulmonary diseases and generating ischaemic/anoxic insults [11]. In the heart, particulate matter-induced inflammation may increase the vulnerability of pre-existing coronary arterial plaques, leading to acute coronary syndrome [12]. Previous findings have suggested that respiratory viral infections may interact with particulate matter, thereby causing additional oxidative stress and expediting inflammation, resulting in cardiac arrest following the exacerbation of respiratory failure/ cardiac complications $[10,13] . \mathrm{PM}_{2.5}$ may have a common effect on the occurrence of respiratory/cardiac origin OHCA in patients with underlying cardiopulmonary diseases.

Most patients with respiratory origin OHCA developed non-shockable rhythms as the initial cardiac rhythm. The incremental severity of COPD is associated with an increasing prevalence of non-shockable rhythms [14], indicating that we may have included patients with severe respiratory diseases. We previously reported that the occurrence of an initial non-shockable rhythm was associated with an increase in $\mathrm{PM}_{2.5}$ concentration in cardiac origin OHCAs [5]. Most non- $\mathrm{PM}_{2.5}$ cardiac origin $\mathrm{OHCAs}$ are due to ischaemic heart disease accompanied by an initial shockable rhythm [4]. More than $20 \%$ of cardiac origin OHCA patients are thought to have comorbid COPD [14], which may be influenced by $\mathrm{PM}_{2.5}$ exposure, thus contributing to the manifestation of an initial non-shockable rhythm. Taken together, increased $\mathrm{PM}_{2.5}$ concentrations are associated with respiratory/cardiac origin OHCAs that commonly present with a non-shockable rhythm, which remains a strong predictor of poor outcomes [15].

In conclusion, increased $\mathrm{PM}_{2.5}$ concentration is associated with bystander-witnessed respiratory origin OHCAs. $\mathrm{PM}_{2.5}$-related deterioration of respiratory function and cardiac complications may promote 
OHCA in individuals with pre-existing cardiopulmonary conditions. Our findings emphasise the need to improve air quality, which is one of the sustainable development goals.

Sunao Kojima ${ }^{1}$, Takehiro Michikawa ${ }^{2}$, Kunihiko Matsui ${ }^{3}$, Hisao Ogawa ${ }^{4}$, Shin Yamazaki $^{5}$, Hiroshi Nitta $^{5}$, Akinori Takami ${ }^{6}$, Kayo Ueda ${ }^{7}$, Yoshio Tahara ${ }^{8}$, Naohiro Yonemoto ${ }^{9}$, Hiroshi Nonogi ${ }^{10}$, Ken Nagao ${ }^{11}$, Takanori Ikeda ${ }^{12}$ and Yoshio Kobayashi ${ }^{13}$, for the Japanese Circulation Society With Resuscitation Science Study (JCS-ReSS) Group

${ }^{1}$ Dept of General Internal Medicine 3, Kawasaki Medical School General Medical Center, Okayama, Japan. ${ }^{2}$ Dept of Environmental and Occupational Health, School of Medicine, Toho University, Tokyo, Japan. ${ }^{3}$ Dept of General Medicine, Kumamoto University Hospital, Kumamoto, Japan. ${ }^{4}$ National Cerebral and Cardiovascular Center, Suita, Japan. ${ }^{5}$ Centre for Health and Environmental Risk Research, National Institute for Environmental Studies, Tsukuba, Japan. ${ }^{6}$ Centre for Regional Environmental Research, National Institute for Environmental Studies, Tsukuba, Japan. ${ }^{7}$ Environmental Health Sciences, Kyoto University Graduate School of Global Environmental Studies, Kyoto, Japan. ${ }^{8}$ Dept of Cardiovascular Medicine, National Cerebral and Cardiovascular Center, Suita, Japan. ${ }^{9}$ Dept of Public Health, Juntendo University School of Medicine, Tokyo, Japan. ${ }^{10}$ Faculty of Health Science, Osaka Aoyama University, Mino, Japan. ${ }^{11}$ Dept of Cardiovascular Center, Nihon University Hospital, Tokyo, Japan. ${ }^{12}$ Dept of Cardiovascular Medicine, Toho University Faculty of Medicine, Tokyo, Japan. ${ }^{13}$ Dept of Cardiovascular Medicine, Chiba University Graduate School of Medicine, Chiba, Japan.

Correspondence: Sunao Kojima, Dept of General Internal Medicine 3, Kawasaki Medical School General Medical Centre, 2-6-1 Nakasange, Kita-ku, Okayama 700-8505, Japan. E-mail: kojimas@med.kawasaki-m.ac.jp

Received: 14 Nov 2020 | Accepted: 31 Jan 2021

Acknowledgements: Takashi Amano and Kanako Kojima provided administrative assistance to the Subcommittee on Resuscitation Science (Japanese Circulation Society). Staff at the Fire and Disaster Management Agency and the Institute for Fire Safety and Disaster Preparedness of Japan cooperated in establishing and maintaining the All-Japan Utstein Registry. These individuals received no additional compensation, outside of their usual salary, for their contributions. We thank the emergency medical services personnel and physicians in Japan.

Conflict of Interest: S. Kojima reports grants and personal fees from Teijin Pharma, Ltd, grants from Daiichi Sankyo Company, Ltd, Chugai Pharmaceutical Company, Ltd and Bayer Yakuhin, Ltd, outside the submitted work. T. Michikawa has nothing to disclose. K. Matsui has nothing to disclose. H. Ogawa reports personal fees from Bayer Yakuhin, Novartis Pharma, Bristol-Meyers Squibb, Pfizer and Towa Pharmaceutical, outside the submitted work. S. Yamazaki has nothing to disclose. H. Nitta has nothing to disclose. A. Takami reports grants and personal fees from Ministry of Environment, Japan, during the conduct of the study; personal fees from Sophia University, Automobile Research Institute and University of Occupational and Environmental Health, outside the submitted work. K. Ueda has nothing to disclose. Y. Tahara has nothing to disclose. N. Yonemoto has nothing to disclose. H. Nonogi has nothing to disclose. K. Nagao has nothing to disclose. T. Ikeda has nothing to disclose. Y. Kobayashi reports grants from Takeda Pharmaceutical, Astellas, Ingelheim, Lifeline, Nipro, Otsuka Pharmaceutical, Terumo and Win International, grants and personal fees from Abbott Medical Japan, Boehringer Ingelheim and Daiichi-Sankyo, and personal fees from Bayer and Bristol-Myers Squibb, outside the submitted work.

Support statement: This study was supported by the Environment Research and Technology Development Fund (Ministry of Environment, Japan) (5-1751) and Kawasaki Medical School (Japan) (R02 B-100). The funding sources had no role in the design and conduct of the study; collection, management, analysis, and interpretation of the data; preparation, review, or approval of the manuscript; and decision to submit the manuscript for publication. Funding information for this article has been deposited with the Crossref Funder Registry.

\section{References}

1 Cohen AJ, Brauer M, Burnett R, et al. Estimates and 25-year trends of the global burden of disease attributable to ambient air pollution: an analysis of data from the Global Burden of Diseases Study 2015. Lancet 2017; 389: 1907-1918.

2 Zhao R, Chen S, Wang W, et al. The impact of short-term exposure to air pollutants on the onset of out-of-hospital cardiac arrest: a systematic review and meta-analysis. Int J Cardiol 2017; 226: 110-117.

3 Kim JH, Hong J, Jung J, et al. Effect of meteorological factors and air pollutants on out-of-hospital cardiac arrests: a time series analysis. Heart 2020; 106: 1218-1227.

4 Myat A, Song KJ, Rea T. Out-of-hospital cardiac arrest: current concepts. Lancet 2018; 391: 970-979.

5 Kojima S, Michikawa T, Matsui K, et al. Association of fine particulate matter exposure with bystander-witnessed out-of-hospital cardiac arrest of cardiac origin in Japan. JAMA Netw Open 2020; 3: e203043.

6 Jacobs I, Nadkarni V, Bahr J, et al. Cardiac arrest and cardiopulmonary resuscitation outcome reports: update and simplification of the Utstein templates for resuscitation registries. A statement for healthcare professionals from a task force of the international liaison committee on resuscitation (American Heart Association, European Resuscitation Council, Australian Resuscitation Council, New Zealand Resuscitation Council, Heart and Stroke Foundation of Canada, InterAmerican Heart Foundation, Resuscitation Council of Southern Africa). Resuscitation 2004; 63: 233-249.

7 Janes H, Sheppard L, Lumley T. Case-crossover analyses of air pollution exposure data: referent selection strategies and their implications for bias. Epidemiology 2005; 16: 717-726.

8 Atkinson RW, Kang S, Anderson HR, et al. Epidemiological time series studies of PM2.5 and daily mortality and hospital admissions: a systematic review and meta-analysis. Thorax 2014; 69: 660-665.

9 Ghio AJ, Carraway MS, Madden MC. Composition of air pollution particles and oxidative stress in cells, tissues, and living systems. J Toxicol Environ Health B 2012; 15: 1-21. 
10 MacNee W, Donaldson K. Mechanism of lung injury caused by PM10 and ultrafine particles with special reference to COPD. Eur Respir J Suppl 2003; 40: 47s-51s.

11 Dominici F, Peng RD, Bell ML, et al. Fine particulate air pollution and hospital admission for cardiovascular and respiratory diseases. JAMA 2006; 295: 1127-1134.

12 Geng J, Liu H, Ge P, et al. PM2.5 promotes plaque vulnerability at different stages of atherosclerosis and the formation of foam cells via TLR4/MyD88/NFKB pathway. Ecotoxicol Environ Saf 2019; 176: 76-84.

13 Ciencewicki J, Jaspers I. Air pollution and respiratory viral infection. Inhal Toxicol 2007; 19: 1135-1146.

14 Granfeldt A, Wissenberg M, Hansen SM, et al. Severity of chronic obstructive pulmonary disease and presenting rhythm in patients with out-of-hospital cardiac arrest. Resuscitation 2018; 126: 111-117.

15 Martinell L, Nielsen N, Herlitz J, et al. Early predictors of poor outcome after out-of-hospital cardiac arrest. Crit Care 2017; 21: 96

Copyright (C)The authors 2021. This version is distributed under the terms of the Creative Commons Attribution Non-Commercial Licence 4.0. For commercial reproduction rights and permissions contact permissionsdersnet.org 